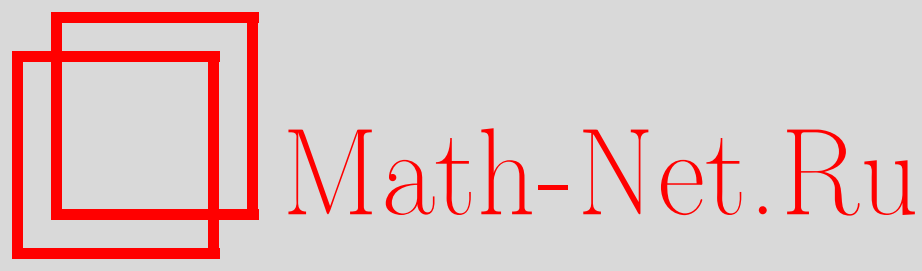

Х. Ваджахат А. Риаз, М. Хассан, Модель обобщенного магнетика Гейзенберга на решетке и ее квазидетерминантные солитонные решения, ТМФ, 2018, том 195, номер 2, 197-208

DOI: https://doi.org/10.4213/tmf9437

Использование Общероссийского математического портала Math-Net.Ru подразумевает, что вы прочитали и согласны с пользовательским соглашением http://www . mathnet.ru/rus/agreement

Параметры загрузки:

IP : 3.85 .5 .30

26 апреля 2023 г., 14:09:45

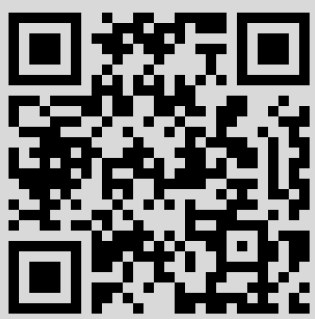


Том 195, № 2

май, 2018

(C) 2018 г

Х. Ваджахат А. Риаз*, М. Хассан*

\title{
МОДЕЛЬ ОБОБЩЕННОГО МАГНЕТИКА ГЕЙЗЕНБЕРГА НА РЕШЕТКЕ \\ И ЕЕ КВАЗИДЕТЕРМИНАНТНЫЕ СОЛИТОННЫЕ РЕШЕНИЯ
}

\begin{abstract}
Рассмотрено преобразование Дарбу обобщенной решеточной (или полудискретной) модели магнетика Гейзенберга. Введены преобразования Дарбу на решениях пары Лакса и на решениях спинового эволюционного уравнения модели обобщенного магнетика Гейзенберга на решетке. Решения выражаются через квазидетерминанты. Приведено общее выражение для $K$-солитонного решения в терминах квазидетерминантов. С использованием свойств квазидетерминантов построены одно- и двухсолитонные решения модели обобщенного магнетика Гейзенберга на решетке.
\end{abstract}

Ключевые слова: дискретные интегрируемые системы, солитоны, преобразование Дарбу.

DOI: https://doi.org/10.4213/tmf9437

\section{1. ВВЕДЕНИЕ}

Наблюдается возрастающий интерес к изучению непрерывных и решеточных моделей магнетика Гейзенберга [1]-[22]. Модель непрерывного магнетика Гейзенберга является полностью интегрируемой системой, и спиновое эволюционное уравнение для него имеет точные решения, называемые солитонами. Эта модель несет много признаков интегрируемости, такие как наличие бесконечного числа сохраняющихся величин (локальных и нелокальных), представление Лакса и свойства Пенлеве. Солитонные решения изучались методом обратной задачи рассеяния, с помощью преобразования Беклунда, преобразования Дарбу и с использованием других методов построения решений (см., например, [1]-[3], [15]-[21]).

Как и для непрерывной модели, модель магнетика Гейзенберга на решетке сохраняет интегрируемость системы. Модель магнетика Гейзенберга на решетке предложена во многих работах (см., например, [5]-[8]). Полная интегрируемость модели

${ }^{*}$ Department of Physics, University of Punjab, Lahore, Pakistan.

E-mail: ahmed.phyy@gmail.com, mhassan.physics@pu.edu.pk 
магнетика Гейзенберга на решетке проявляется в существовании пары Лакса, высших симметрий, преобразования Беклунда и обратной задачи рассеяния [2], [5]-[14].

В настоящей работе вводится квазидетерминантная матрица Дарбу, которая действует на решениях пары Лакса модели обобщенного магнетика Гейзенберга на решетке (ОМГР) и порождает солитонные решения этой модели. Получено общее выражение для $K$-солитонного решения в терминах квазидетерминантов. Наконец, используя свойства квазидетерминантов, мы получили явное выражение для односолитонного решения в общем $(N \times N)$-случае и простейшем случае $N=2$ модели ОМГР.

\section{2. МОДЕЛЬ ОМГР}

Пара Лакса для модели ОМГР [14] имеет вид

$$
\begin{aligned}
\Psi_{n+1} & =A_{n} \Psi_{n}, & A_{n} & =I+\lambda U_{n}, \\
\frac{d}{d t} \Psi_{n} & =B_{n} \Psi_{n}, & B_{n} & =\frac{\lambda}{1-\lambda^{2}} J_{n}+\frac{\lambda^{2}}{1-\lambda^{2}} J_{n} U_{n},
\end{aligned}
$$

где $\Psi_{n} \equiv \Psi_{n}(\lambda, t)$ - собственная $(N \times N)$-матрица. Матричная функция $U_{n} \equiv U_{n}(t)$ также является $(N \times N)$-матрицей, причем на нее налагаются условия $U_{n}^{2}=I$ и $J_{n} U_{n}=U_{n-1} J_{n}$. Последнее условие означает $J_{n} A_{n}=A_{n-1} J_{n}$. Пара Лакса (1) удовлетворяет условию совместности $d A_{n} / d t+A_{n} B_{n}-B_{n+1} A_{n}=0$ для любого собственного числа $\lambda$, если матричнозначная функция $U_{n}$ удовлетворяет уравнению движения

$$
\frac{d}{d t} U_{n}=J_{n+1}-J_{n}
$$

Из уравнения (2) следует

$$
J_{n+1}\left(U_{n+1}+U_{n}\right)=\left(U_{n}+U_{n-1}\right) J_{n} .
$$

Уравнение (3) удовлетворяется, если положить

$$
J_{n}=2 i a U_{n-1}\left(U_{n}+U_{n-1}\right)^{-1}+2 b\left(U_{n}+U_{n-1}\right)^{-1} .
$$

Подставляя это выражение в уравнение (2), получим

$$
\frac{d}{d t} U_{n}=\Delta_{n}\left[2 i a U_{n-1}\left(U_{n}+U_{n-1}\right)^{-1}+2 b\left(U_{n}+U_{n-1}\right)^{-1}\right]
$$

где $\Delta_{n} f_{n}=f_{n+1}-f_{n}$. При $N=2$ имеем простейший $(2 \times 2)$-случай, для которого матрицу $U_{n}$ можно представить в виде $U_{n}=U_{n}^{a} \sigma_{a}$, где $\sigma_{a}$ - известные матрицы Паули, а условие на матрицу $U_{n}$ принимает вид $U_{n}^{2}=I, I-$ единичная $(2 \times 2)$-матрица. Подставим $2\left(U_{n}+U_{n-1}\right)^{-1}=\left(U_{n}+U_{n-1}\right) /\left(1+\mathbf{U}_{n} \cdot \mathbf{U}_{n-1}\right)$ в (4), тогда уравнение движения в векторных обозначениях можно представить в виде

$$
\frac{d}{d t} \mathbf{U}_{n}=\Delta_{n}\left[a \frac{\mathbf{U}_{n} \times \mathbf{U}_{n-1}}{1+\mathbf{U}_{n} \cdot \mathbf{U}_{n-1}}+b \frac{\mathbf{U}_{n}+\mathbf{U}_{n-1}}{1+\mathbf{U}_{n} \cdot \mathbf{U}_{n-1}}\right], \quad \mathbf{U}_{n}^{2}=1
$$


В следующем разделе мы введем преобразование Дарбу на решении $\Psi_{n}$ пары Лакса (1) и на решении $U_{n}$ уравнения движения (2) модели ОМГР. Преобразование Дарбу является одним из важных методов построения решений нелинейных интегрируемых систем. Этот метод успешно использовался для одномерных и многомерных интегрируемых систем. Его обобщили на многие другие приложения, также получены решения интегрируемых уравнений в виде квазидетерминантов, граммианов и вронскианов [23]-[27].

\section{3. ПРЕОБРАЗОВАНИЕ ДАРБУ}

Напишем новое решение $\Psi_{n}[1]$ для пары Лакса $(1)$, полученное из старого решения $\Psi_{n}$ с помощью $(N \times N)$-матрицы Дарбу $D_{n}(\lambda)=D_{n}(t, \lambda)$, тем самым преобразование Дарбу на матричном решении $\Psi_{n}$ задается выражением

$$
\Psi_{n}[1]=D_{n}(\lambda) \Psi_{n}
$$

где $(N \times N)$-матрицу $D_{n}(\lambda)$ можно определить. Ковариантность пары Лакса относительно преобразования Дарбу требует, чтобы новое решение $\Psi_{n}[1]$ удовлетворяло паре Лакса (1) и имело вид

$$
\begin{aligned}
\Psi_{n+1}[1] & =A_{n}[1] \Psi_{n}[1], & A_{n}[1] & =I+\lambda U_{n}[1], \\
\frac{d}{d t} \Psi_{n}[1] & =B_{n}[1] \Psi_{n}[1], & B_{n}[1] & =\frac{\lambda}{1-\lambda^{2}} J_{n}[1]+\frac{\lambda^{2}}{1-\lambda^{2}} J_{n}[1] U_{n}[1]
\end{aligned}
$$

В нашем случае выберем матрицу Дарбу вида

$$
D_{n}(\lambda)=\lambda^{-1} I-\mathcal{Q}_{n},
$$

где $\mathcal{Q}_{n}-(N \times N)$-матрица, а $I$ - тождественная $(N \times N)$-матрица. После подстановки выражения для $\Psi_{n}[1](6)$ в (7) коэффициент при $\lambda$ будет преобразованием Дарбу матричных функций $U_{n}$ и $J_{n}$ :

$$
U_{n}[1]=U_{n}-\left(\mathcal{Q}_{n+1}-\mathcal{Q}_{n}\right), \quad J_{n}[1]=J_{n}-\frac{d}{d t} \mathcal{Q}_{n}
$$

а матрица $\mathcal{Q}_{n}$ должна удовлетворять уравнениям

$$
\begin{gathered}
\left(\mathcal{Q}_{n+1}-\mathcal{Q}_{n}\right) \mathcal{Q}_{n}=U_{n} \mathcal{Q}_{n}-\mathcal{Q}_{n+1} U_{n} \\
\frac{d \mathcal{Q}_{n}}{d t}\left(I-\mathcal{Q}_{n}^{2}\right)=\left[\mathcal{Q}_{n}, J_{n}\left(\mathcal{Q}_{n}+U_{n}\right)\right]
\end{gathered}
$$

Найдем теперь явное выражение для матрицы $\mathcal{Q}_{n}$, которая одновременно удовлетворяет уравнениям (10), (11). Явное выражение можно получить в терминах частных векторных решений пары Лакса модели ОМГР. Заметим, что указанная в работе [14] дискретизация и полученное в результате преобразование Беклунда можно вывести из системы (8), (9), исключив из нее волновые функции.

Возьмем $N$ различных параметров (действительных или комплексных) $\lambda_{i}\left(\lambda_{i} \neq \lambda_{j}\right)$, $i=1,2, \ldots, N, j=i+1, i+2, \ldots, i+N$. Для каждого значения $\lambda_{i}$ определим $N$ 
независимых собственных векторов - решений $\Psi_{n}\left(\lambda_{i}\right)\left|e_{i}\right\rangle$ пары Лакса $(1)$ - и по этим собственным векторам построим обратимые $(N \times N)$-матрицы $\mathcal{H}_{n}$ вида

$$
\mathcal{H}_{n}=\left(\Psi_{n}\left(\lambda_{1}\right)\left|e_{1}\right\rangle, \ldots, \Psi_{n}\left(\lambda_{N}\right)\left|e_{N}\right\rangle\right)=\left(\left|h_{1}\right\rangle_{n}, \ldots,\left|h_{N}\right\rangle_{n}\right)
$$

В этом случае каждый собственный вектор $\left|h_{i}\right\rangle_{n}=\Psi_{n}\left(\lambda_{i}\right)\left|e_{i}\right\rangle$ матрицы $\mathcal{H}_{n}$ является решением (столбцом) пары Лакса (1), т. е.

$$
\begin{aligned}
\left|h_{i}\right\rangle_{n+1} & =\left|h_{i}\right\rangle_{n}+\lambda_{i} U_{n}\left|h_{i}\right\rangle_{n} \\
\frac{d}{d t}\left|h_{i}\right\rangle_{n} & =\frac{\lambda_{i}}{1-\lambda_{i}^{2}} J_{n}\left|h_{i}\right\rangle_{n}+\frac{\lambda_{i}^{2}}{1-\lambda_{i}^{2}} J_{n} U_{n, i}\left|h_{i}\right\rangle_{n} .
\end{aligned}
$$

Частное матричное решение $\mathcal{H}_{n}$ удовлетворяет паре Лакса. Возьмем обратимую $(N \times N)$-матрицу $\Lambda$ с собственными числами $\lambda_{i}$ на диагонали $\Lambda=\operatorname{diag}\left(\lambda_{1}, \ldots, \lambda_{N}\right)$ и представим пару Лакса (13) в виде

$$
\begin{aligned}
\mathcal{H}_{n+1} & =\mathcal{H}_{n}+U_{n} \mathcal{H}_{n} \Lambda, \\
\frac{d}{d t} \mathcal{H}_{n} & =J_{n} \mathcal{H}_{n} \Lambda\left(I-\Lambda^{2}\right)^{-1}+J_{n} U_{n} \mathcal{H}_{n} \Lambda^{2}\left(I-\Lambda^{2}\right)^{-1},
\end{aligned}
$$

где $\mathcal{H}_{n}$ - частное матричное решение пары Лакса (1). Введем матрицу $\mathcal{Q}_{n}$ с помощью частного матричного решения $\mathcal{H}_{n}$, а именно $\mathcal{Q}_{n}=\mathcal{H}_{n} \Lambda^{-1} \mathcal{H}_{n}^{-1}$. Покажем теперь, что матрица $\mathcal{Q}_{n}$ удовлетворяет системе уравнений $(10),(11)$. Чтобы проверить уравнение (10), применим разностный оператор $\Delta_{n}$ сдвига вперед к матрице $\mathcal{Q}_{n}$ и проведем вычисления:

$$
\begin{aligned}
\left(\Delta_{n}\right. & \left.\mathcal{Q}_{n}\right) \mathcal{Q}_{n}=\left(\mathcal{Q}_{n+1}-\mathcal{Q}_{n}\right) \mathcal{Q}_{n}= \\
& =\left(\mathcal{H}_{n+1} \Lambda^{-1} \mathcal{H}_{n+1}^{-1}-\mathcal{H}_{n} \Lambda^{-1} \mathcal{H}_{n}^{-1}\right) \mathcal{H}_{n} \Lambda^{-1} \mathcal{H}_{n}^{-1}= \\
& =\mathcal{H}_{n+1} \Lambda^{-1} \mathcal{H}_{n+1}^{-1} \mathcal{H}_{n} \Lambda^{-1} \mathcal{H}_{n}^{-1}-\mathcal{H}_{n} \Lambda^{-2} \mathcal{H}_{n}^{-1}+\mathcal{H}_{n+1} \Lambda^{-2} \mathcal{H}_{n}^{-1}-\mathcal{H}_{n+1} \Lambda^{-2} \mathcal{H}_{n}^{-1}= \\
& =\left(\mathcal{H}_{n+1}-\mathcal{H}_{n}\right) \Lambda^{-1} \mathcal{H}_{n}^{-1} \mathcal{H}_{n} \Lambda^{-1} \mathcal{H}_{n}^{-1}-\mathcal{H}_{n+1} \Lambda^{-1} \mathcal{H}_{n+1}^{-1}\left(\mathcal{H}_{n+1}-\mathcal{H}_{n}\right) \Lambda^{-1} \mathcal{H}_{n}^{-1}= \\
& =U_{n} \mathcal{Q}_{n}-\mathcal{Q}_{n+1} U_{n}
\end{aligned}
$$

что совпадает с уравнением (10). Чтобы проверить уравнение (11), подействуем оператором $d / d t$ на матрицу $\mathcal{Q}_{n}$ и проведем следующие вычисления:

$$
\begin{aligned}
\frac{d \mathcal{Q}_{n}}{d t} & \left(I-\mathcal{Q}_{n}^{2}\right)=\left(\frac{d}{d t} \mathcal{H}_{n} \Lambda^{-1} \mathcal{H}_{n}^{-1}\right)\left(I-\mathcal{H}_{n} \Lambda^{-2} \mathcal{H}_{n}^{-1}\right)= \\
= & \frac{d \mathcal{H}_{n}}{d t} \Lambda^{-1}\left(I-\Lambda^{-2}\right) \mathcal{H}_{n}^{-1}-\mathcal{H}_{n} \Lambda^{-1} \mathcal{H}_{n}^{-1} \frac{d \mathcal{H}_{n}}{d t}\left(I-\Lambda^{-2}\right) \mathcal{H}_{n}^{-1}= \\
= & {\left[J_{n} \mathcal{H}_{n} \Lambda\left(I-\Lambda^{2}\right)^{-1}+J_{n} U_{n} \mathcal{H}_{n} \Lambda^{2}\left(I-\Lambda^{2}\right)^{-1}\right] \Lambda^{-1}\left(I-\Lambda^{-2}\right) \mathcal{H}_{n}^{-1}-} \\
& -\mathcal{H}_{n} \Lambda^{-1} \mathcal{H}_{n}^{-1}\left[J_{n} \mathcal{H}_{n} \Lambda\left(I-\Lambda^{2}\right)^{-1}+J_{n} U_{n} \mathcal{H}_{n} \Lambda^{2}\left(I-\Lambda^{2}\right)^{-1}\right]\left(I-\Lambda^{-2}\right) \mathcal{H}_{n}^{-1}= \\
= & {\left[\mathcal{Q}_{n}, J_{n}\left(\mathcal{Q}_{n}+U_{n}\right)\right], }
\end{aligned}
$$

и получим, таким образом, уравнение (11). Поэтому преобразование Дарбу на матричном решении $\Psi_{n}$ пары Лакса $(1)$ и на матричном решении $U_{n}$ уравнения (4) 
можно представить в виде

$$
\begin{aligned}
\Psi_{n}[1] & =\left(\lambda^{-1} I-\mathcal{H}_{n} \Lambda^{-1} \mathcal{H}_{n}^{-1}\right) \Psi_{n}, \\
U_{n}[1] & =U_{n}-\left(\mathcal{H}_{n+1} \Lambda^{-1} \mathcal{H}_{n+1}^{-1}-\mathcal{H}_{n} \Lambda^{-1} \mathcal{H}_{n}^{-1}\right)= \\
& =\mathcal{H}_{n+1} \Lambda^{-1} \mathcal{H}_{n+1}^{-1} U_{n} \mathcal{H}_{n} \Lambda^{-1} \mathcal{H}_{n}^{-1} .
\end{aligned}
$$

На этом этапе можно утверждать, что если $\left(\Psi_{n}, U_{n}\right)$ являются решениями пары Лакса (1) и (4), то $\left(\Psi_{n}[1], U_{n}[1]\right)$ также являются решениями того же уравнения, они получаются действием матрицы Дарбу $D_{n}(\lambda)$ на $\left(\Psi_{n}, U_{n}\right)$. Преобразование Дарбу (17) сохраняет систему (2), (3), а также оно совместно с редукцией (4). Аналогично, применяя матрицу Дарбу $K$ раз к $\left(\Psi_{n}, U_{n}\right)$, мы получим $\left(\Psi_{n}[K], U_{n}[K]\right)$, т. е. $K$-солитонное решение модели ОМГР.

Можно заметить, что матрица Дарбу-Беклунда, построенная из $N$ собственных векторов, факторизуется на элементарные преобразования Дарбу. В стандартном преобразовании Дарбу мы имеем дело с матричным решением пары Лакса, а в элементарном преобразовании Дарбу - с векторным решением пары Лакса.

\section{4. КВАЗИДЕТЕРМИНАНТНЫЕ РЕШЕНИЯ}

Если матрица $\mathcal{H}_{n}$ обратима, то матрицу Дарбу можно выразить через квазидетерминанты. Подробности использования и свойства квазидетерминантов содержатся в работах [28]-[30]. Однократное преобразование Дарбу на матричном решении $\Psi_{n}$ пары Лакса (1) имеет вид

$$
\begin{aligned}
\Psi_{n}[1] & \equiv D_{n}(\lambda ; t) \Psi_{n}=\left(\lambda^{-1} I-\mathcal{H}_{n} \Lambda^{-1} \mathcal{H}_{n}^{-1}\right) \Psi_{n}= \\
& =\left|\begin{array}{cc}
\mathcal{H}_{n} & \Psi_{n} \\
\mathcal{H}_{n} \Lambda^{-1} & \lambda^{-1} \Psi_{n}
\end{array}\right| .
\end{aligned}
$$

Однократное преобразование Дарбу на матрице $U_{n}$ можно представить в виде

$$
\begin{aligned}
U_{n}[1] & =U_{n}-\left(\mathcal{Q}_{n+1}-\mathcal{Q}_{n}\right)=\mathcal{Q}_{n+1} U_{n} \mathcal{Q}_{n}^{-1}= \\
& =\mathcal{H}_{n+1} \Lambda^{-1} \mathcal{H}_{n+1}^{-1} U_{n} \mathcal{H}_{n} \Lambda^{-1} \mathcal{H}_{n}^{-1}= \\
& =\left|\begin{array}{cc}
\mathcal{H}_{n+1} & I \\
\mathcal{H}_{n+1} \Lambda^{-1} & O
\end{array}\right| U_{n}\left|\begin{array}{cc}
\mathcal{H}_{n} & I \\
\mathcal{H}_{n} \Lambda^{-1} & O
\end{array}\right|^{-1},
\end{aligned}
$$

где $O$ - нулевая $(N \times N)$-матрица. Полученные результаты (18), (19) можно расширить и обобщить на $K$-кратное преобразование Дарбу. Чтобы найти $K$-кратное преобразование Дарбу, введем частные матричные решения $\mathcal{H}_{n, k}$ для пары Лакса, соответствующие $\Lambda=\Lambda_{k}, k=1,2, \ldots, K$. Примененное $K$ раз преобразование Дарбу порождает матричное решение $\Psi_{n}[K]$ для пары Лакса (1) и может быть представлено в виде квазидетерминанта:

$$
\Psi_{n}[K]=\left|\begin{array}{ccccc}
\mathcal{H}_{n, 1} & \mathcal{H}_{n, 2} & \cdots & \mathcal{H}_{n, K} & \Psi_{n} \\
\mathcal{H}_{n, 1} \Lambda_{1}^{-1} & \mathcal{H}_{n, 2} \Lambda_{2}^{-1} & \cdots & \mathcal{H}_{n, K} \Lambda_{K}^{-1} & \lambda^{-1} \Psi_{n} \\
\vdots & \vdots & \ddots & \vdots & \vdots \\
\mathcal{H}_{n, 1} \Lambda_{1}^{-(K-1)} & \mathcal{H}_{n, 2} \Lambda_{2}^{-(K-1)} & \ldots & \mathcal{H}_{n, K} \Lambda_{K}^{-(K-1)} & \lambda^{-(K-1)} \Psi_{n} \\
\mathcal{H}_{n, 1} \Lambda_{1}^{-K} & \mathcal{H}_{n, 2} \Lambda_{2}^{-K} & \cdots & \mathcal{H}_{n, K} \Lambda_{K}^{-K} & \lambda^{-K} \Psi_{n}
\end{array}\right| .
$$


Аналогичное квазидетерминантное представление $U_{n}[K]$ имеет вид

$$
\begin{aligned}
& U_{n}[K]=\left|\begin{array}{ccccc}
\mathcal{H}_{n+1,1} & \mathcal{H}_{n+1,2} & \cdots & \mathcal{H}_{n+1, K} & I \\
\mathcal{H}_{n+1,1} \Lambda_{1}^{-1} & \mathcal{H}_{n+1,2} \Lambda_{2}^{-1} & \cdots & \mathcal{H}_{n+1, K} \Lambda_{K}^{-1} & O \\
\vdots & \vdots & \ddots & \vdots & \vdots \\
\mathcal{H}_{n+1,1} \Lambda_{1}^{-(K-1)} & \mathcal{H}_{n+1,2} \Lambda_{2}^{-(K-1)} & \ldots & \mathcal{H}_{n+1, K} \Lambda_{K}^{-(K-1)} & O \\
\mathcal{H}_{n+1,1} \Lambda_{1}^{-K} & \mathcal{H}_{n+1,2} \Lambda_{2}^{-K} & \cdots & \mathcal{H}_{n+1, K} \Lambda_{K}^{-K} & O
\end{array}\right| \times \\
& \times U_{n}\left|\begin{array}{ccccc}
\mathcal{H}_{n, 1} & \mathcal{H}_{n, 2} & \cdots & \mathcal{H}_{n, K} & I \\
\mathcal{H}_{n, 1} \Lambda_{1}^{-1} & \mathcal{H}_{n, 2} \Lambda_{2}^{-1} & \cdots & \mathcal{H}_{n, K} \Lambda_{K}^{-1} & O \\
\vdots & \vdots & \ddots & \vdots & \vdots \\
\mathcal{H}_{n, 1} \Lambda_{1}^{-(K-1)} & \mathcal{H}_{n, 2} \Lambda_{2}^{-(K-1)} & \cdots & \mathcal{H}_{n, K} \Lambda_{K}^{-(K-1)} & O \\
\mathcal{H}_{n, 1} \Lambda_{1}^{-K} & \mathcal{H}_{n, 2} \Lambda_{2}^{-K} & \cdots & \mathcal{H}_{n, K} \Lambda_{K}^{-K} & O
\end{array}\right|^{-1}
\end{aligned}
$$

Выражения (20) и (21) представляют собой соответственно квазидетерминантное решение $\Psi_{n}[K]$ пары Лакса и квазидетерминантное решение $U_{n}[K]$ модели ОМГР. Полученные результаты легко доказать методом индукции.

\section{5. ЯВНЫЕ РЕШЕНИЯ}

В настоящем разделе получено солитонное решение из затравочного (тривиального) решения с помощью решения пары Лакса модели ОМГР. Для этого перепишем матрицу $\mathcal{Q}_{n}^{(K)}$ из $(21)$ в следующем более удобном виде:

$$
\mathcal{Q}_{n}^{(K)}=\left|\begin{array}{ll}
\mathcal{H}_{n} & \mathcal{I}^{(K)} \\
\widetilde{\mathcal{H}}_{n} & O
\end{array}\right|,
$$

где $\mathcal{I}^{(K)}, \widetilde{\mathcal{H}}_{n}$ и $\mathcal{H}_{n}$ - матрицы размера $(N K \times N),(N \times N K)$ и $(N K \times N K)$ соответственно. Эти матрицы имеют вид

$$
\begin{aligned}
\mathcal{I}^{(K)} & =\left(\begin{array}{lllll}
I & O & \ldots & O
\end{array}\right)^{\mathrm{T}}, \\
\widetilde{\mathcal{H}}_{n} & =\left(\begin{array}{llll}
\mathcal{H}_{n, 1} \Lambda_{1}^{-K} & \mathcal{H}_{n, 2} \Lambda_{2}^{-K} & \ldots & \mathcal{H}_{n, K} \Lambda_{K}^{-K}
\end{array}\right), \\
\mathcal{H}_{n} & =\left(\begin{array}{cccc}
\mathcal{H}_{n, 1} & \mathcal{H}_{n, 2} & \ldots & \mathcal{H}_{n, K} \\
\mathcal{H}_{n, 1} \Lambda_{1}^{-1} & \mathcal{H}_{n, 2} \Lambda_{2}^{-1} & \ldots & \mathcal{H}_{n, K} \Lambda_{K}^{-1} \\
\vdots & \vdots & \ddots & \vdots \\
\mathcal{H}_{n, 1} \Lambda_{1}^{-(K-1)} & \mathcal{H}_{n, 2} \Lambda_{2}^{-(K-1)} & \ldots & \mathcal{H}_{n, K} \Lambda_{K}^{-(K-1)}
\end{array}\right) .
\end{aligned}
$$

Элементы матрицы $\mathcal{Q}_{n}^{(K)}$ можно представить в виде

$$
\begin{aligned}
\mathcal{Q}_{n, i j}^{(K)} & =\left(\begin{array}{cc}
\mid \mathcal{H}_{n} & I^{(K)} \\
\widetilde{\mathcal{H}}_{n} & O
\end{array} \mid\right)_{i j}=\left|\begin{array}{cc}
\mathcal{H}_{n} & I_{j}^{(K)} \\
\left(\widetilde{\mathcal{H}}_{n}\right)_{i} & 0
\end{array}\right|= \\
& =(-1)^{i+j} \frac{\operatorname{det}\left(\mathcal{H}_{n}\right)_{i j}}{\operatorname{det}\left(\mathcal{H}_{n}\right)}, \quad i, j=1,2, \ldots, K,
\end{aligned}
$$

где $\left(\widetilde{\mathcal{H}}_{n}\right)_{i}, \mathcal{I}_{j}^{(K)}$ являются $i$-й строкой и $j$-м столбцом матриц $\widetilde{\mathcal{H}}_{n}, \mathcal{I}^{(K)}$ соответственно. Общую формулу (24) можно использовать для явного построения систем, 
имеющих матричные пары Лакса различных размерностей. В простейшем случае $N=2$ матрица $\mathcal{Q}_{n}^{(K)}$ имеет следующее представление:

$$
\mathcal{Q}_{n}^{(K)} \equiv\left(\begin{array}{ll}
\mathcal{Q}_{n, 11}^{(K)} & \mathcal{Q}_{n, 12}^{(K)} \\
\mathcal{Q}_{n, 21}^{(K)} & \mathcal{Q}_{n, 22}^{(K)}
\end{array}\right)=\left|\begin{array}{ll}
\mathcal{H}_{n} & \mathcal{I}^{(K)} \\
\widetilde{\mathcal{H}}_{n} & O_{2}
\end{array}\right|
$$

a ее элементы имеют вид

$$
\mathcal{Q}_{n, i j}^{(K)}=\left|\begin{array}{cc}
\mathcal{H}_{n} & \mathcal{I}_{j}^{(K)} \\
\left(\widetilde{\mathcal{H}}_{n}\right)_{i} & 0
\end{array}\right|=(-1)^{i+j} \frac{\operatorname{det}\left(\mathcal{H}_{n}\right)_{i j}}{\operatorname{det}\left(\mathcal{H}_{n}\right)}, \quad i, j=1,2 .
$$

Для односолитонного решения $(K=1)$ имеем

$$
\begin{gathered}
\mathcal{I}^{(1)}=I_{2}=\left(\begin{array}{ll}
1 & 0 \\
0 & 1
\end{array}\right), \\
\mathcal{H}_{n, 1}=\left(\begin{array}{ll}
h_{n, 11}^{(1)} & h_{n, 12}^{(2)} \\
h_{n, 21}^{(1)} & h_{n, 22}^{(2)}
\end{array}\right), \quad \Lambda_{1}=\left(\begin{array}{cc}
\eta_{1} & 0 \\
0 & \bar{\eta}_{1}
\end{array}\right), \\
\widetilde{\mathcal{H}}_{n}=\mathcal{H}_{n, 1} \Lambda_{1}^{-1}= \\
=\left(\begin{array}{ll}
h_{n, 11}^{(1)} & h_{n, 12}^{(2)} \\
h_{n, 21}^{(1)} & h_{n, 22}^{(2)}
\end{array}\right)\left(\begin{array}{cc}
\eta_{1}^{-1} & 0 \\
0 & \bar{\eta}_{1}^{-1}
\end{array}\right)=\left(\begin{array}{ll}
\eta_{1}^{-1} h_{n, 11}^{(1)} & \bar{\eta}_{1}^{-1} h_{n, 12}^{(2)} \\
\eta_{1}^{-1} h_{n, 21}^{(1)} & \bar{\eta}_{1}^{-1} h_{n, 22}^{(2)}
\end{array}\right) .
\end{gathered}
$$

Подставляя $(27)$ в $(26)$, получим матричный элемент $\mathcal{Q}_{n, 12}^{(1)}$ матрицы $\mathcal{Q}_{n}^{(1)}$ в виде

$$
\begin{aligned}
\mathcal{Q}_{n, 12}^{(1)} & =\left|\begin{array}{cc}
\mathcal{H}_{n} & \mathcal{I}_{2}^{(1)} \\
\left(\widetilde{\mathcal{H}}_{n}\right)_{1} & O_{2}
\end{array}\right|=\left|\begin{array}{ccc}
h_{n, 11}^{(1)} & h_{n, 12}^{(2)} & 0 \\
h_{n, 21}^{(1)} & h_{n, 22}^{(2)} & 1 \\
\eta_{1}^{-1} h_{n, 11}^{(1)} & \bar{\eta}_{1}^{-1} h_{n, 12}^{(2)} & 0
\end{array}\right|= \\
& =(-1)^{1+2} \frac{\operatorname{det}\left(\begin{array}{cc}
h_{n, 11}^{(1)} & h_{n, 12}^{(2)} \\
\eta_{1}^{-1} h_{n, 11}^{(1)} & \bar{\eta}_{1}^{-1} h_{n, 12}^{(2)}
\end{array}\right)}{\operatorname{det}\left(\begin{array}{ll}
h_{n, 11}^{(1)} & h_{n, 12}^{(2)} \\
h_{n, 21}^{(1)} & h_{n, 22}^{(2)}
\end{array}\right)}=\frac{\left(\eta_{1}^{-1}-\bar{\eta}_{1}^{-1}\right) h_{n, 11}^{(1)} h_{n, 12}^{(2)}}{h_{n, 11}^{(1)} h_{n, 22}^{(2)}-h_{n, 12}^{(2)} h_{n, 21}^{(1)}} .
\end{aligned}
$$

Точно так же получаем

$$
\begin{aligned}
\mathcal{Q}_{n, 21}^{(1)} & =\left|\begin{array}{cc}
\mathcal{H}_{n} & \mathcal{I}_{1}^{(1)} \\
\left(\widetilde{\mathcal{H}}_{n}\right)_{2} & O_{2}
\end{array}\right|=\left|\begin{array}{ccc}
h_{n, 11}^{(1)} & h_{n, 12}^{(2)} & 1 \\
h_{n, 21}^{(1)} & h_{n, 22}^{(2)} & 0 \\
\eta_{1}^{-1} h_{n, 21}^{(1)} & \bar{\eta}_{1}^{-1} h_{n, 22}^{(2)} & 0
\end{array}\right|= \\
& =(-1)^{2+1} \frac{\operatorname{det}\left(\begin{array}{cc}
\eta_{1}^{-1} h_{n, 21}^{(1)} & \bar{\eta}_{1}^{-1} h_{n, 22}^{(2)} \\
h_{n, 21}^{(1)} & h_{n, 22}^{(2)}
\end{array}\right)}{\operatorname{det}\left(\begin{array}{ll}
h_{n, 11}^{(1)} & h_{n, 12}^{(2)} \\
h_{n, 21}^{(1)} & h_{n, 22}^{(2)}
\end{array}\right)}=-\frac{\left(\eta_{1}^{-1}-\bar{\eta}_{1}^{-1}\right) h_{n, 21}^{(1)} h_{n, 22}^{(2)}}{h_{n, 11}^{(1)} h_{n, 22}^{(2)}-h_{n, 12}^{(2)} h_{n, 21}^{(1)}}
\end{aligned}
$$


Аналогично,

$$
\begin{aligned}
\mathcal{Q}_{n, 11}^{(1)} & =\frac{\eta_{1}^{-1} h_{n, 11}^{(1)} h_{n, 22}^{(2)}+\bar{\eta}_{1}^{-1} h_{n, 12}^{(2)} h_{n, 21}^{(1)}}{h_{n, 11}^{(1)} h_{n, 22}^{(2)}-h_{n, 12}^{(2)} h_{n, 21}^{(1)}}, \\
\mathcal{Q}_{n, 22}^{(1)} & =-\frac{\eta_{1}^{-1} h_{n, 11}^{(1)} h_{n, 22}^{(2)}+\bar{\eta}_{1}^{-1} h_{n, 12}^{(2)} h_{n, 21}^{(1)}}{h_{n, 11}^{(1)} h_{n, 22}^{(2)}-h_{n, 12}^{(2)} h_{n, 21}^{(1)}} .
\end{aligned}
$$

Чтобы получить явное выражение для солитонного решения в общем $(N \times N)$-матричном случае, возьмем затравочное решение вида

$$
U_{0} \equiv U_{n}=i\left(\begin{array}{ccc}
c_{1} & & \\
& \ddots & \\
& & c_{N}
\end{array}\right), \quad a=0, \quad b=1
$$

где $c_{i}$ - действительные постоянные, причем $\operatorname{Tr} U_{n}=0$. Тогда простое вычисление приводит к матричному решению $\Psi_{n}$ пары Лакса $(1)$ в следующем виде:

$$
\Psi_{n}=\left(\begin{array}{cc}
\Xi_{n}(\lambda)_{p} & O \\
O & \Xi_{n}(\lambda)_{N-p}
\end{array}\right)
$$

где

$$
\Xi_{n}(\lambda)_{p}=\left(\begin{array}{ccc}
\xi_{n}(\lambda)_{1} & & \\
& \ddots & \\
& & \xi_{n}(\lambda)_{p}
\end{array}\right), \quad \Xi_{n}(\lambda)_{N-p}=\left(\begin{array}{lll}
\xi_{n}(\lambda)_{p+1} & & \\
& \ddots & \\
& & \xi_{n}(\lambda)_{N}
\end{array}\right)
$$

- соответственно $(p \times p)$ - и $(N-p) \times(N-p))$-матрицы. Здесь нижний индекс $n$ является дискретным индексом и

$$
\xi(\lambda)=\left(1+i c_{i} \lambda\right)^{n} \exp \left[\frac{-i c_{i}^{-1} \lambda}{1-\lambda^{2}}+\frac{\lambda^{2}}{1-\lambda^{2}}\right] t .
$$

В случае $N=2$ возьмем затравочное решение вида

$$
U_{0} \equiv U_{n}=i\left(\begin{array}{cc}
c & \\
& -c
\end{array}\right), \quad a=0, \quad b=1,
$$

тогда простые расчеты приводят к следующему матричному решению $\Psi_{n}$ пары Лакca (1):

$$
\Psi_{n}=\left(\begin{array}{ll}
\xi_{n}(\lambda) & \\
& \bar{\xi}_{n}(\lambda)
\end{array}\right)
$$

где

$$
\xi_{n}(\lambda)=(1+i c \lambda)^{n} \exp \left[\frac{-i c^{-1} \lambda}{1-\lambda^{2}}+\frac{\lambda^{2}}{1-\lambda^{2}}\right] t .
$$

$(2 \times 2)$-Матричное решение $\mathcal{H}_{n}$ при $\Lambda=\left(\begin{array}{ll}\eta & 0 \\ 0 & \bar{\eta}\end{array}\right)$ имеет вид

$$
\mathcal{H}_{n}=\left(\Psi_{n}(\eta)|1\rangle, \Psi_{n}(\bar{\eta})|2\rangle\right)=\left(\begin{array}{rr}
\xi(\eta) & -\xi(\bar{\eta}) \\
\bar{\xi}(\eta) & \bar{\xi}(\bar{\eta})
\end{array}\right)
$$




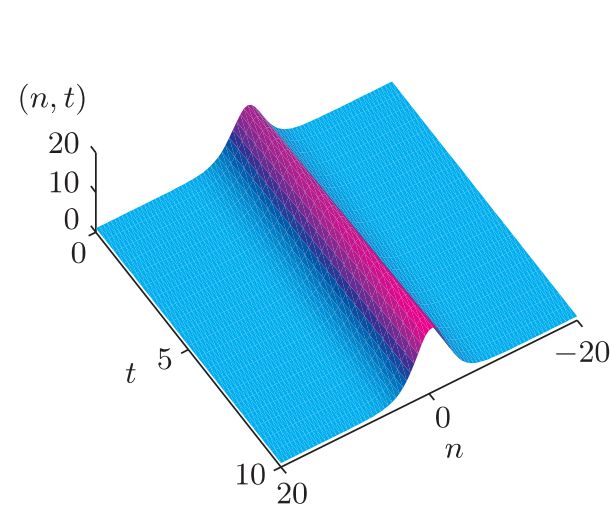

a

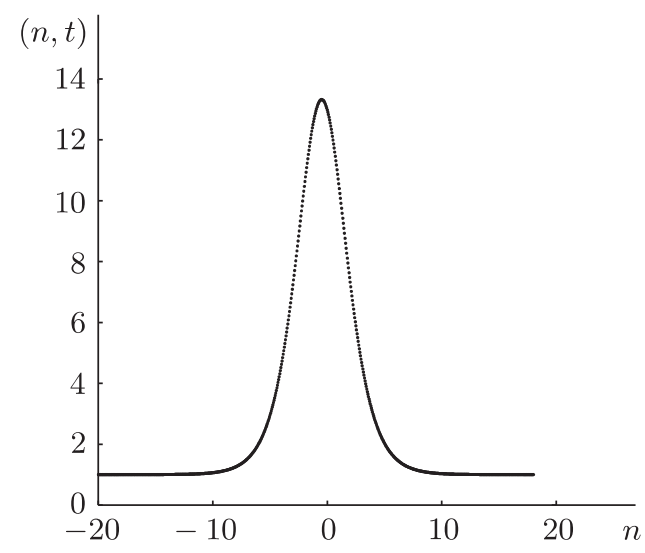

6

Рис. 1. Полудискретное односолитонное решение $U_{n}^{+}$; трехмерное представление (а) и двумерное представление (б) для численных значений $c=-6$, $\eta=-0.029 i$.

Подставляя матрицу $\mathcal{H}_{n}$ в уравнение $(25)$, получим искомое выражение для матрицы $\mathcal{Q}_{n}^{(1)}$ :

$$
\mathcal{Q}_{n}^{(1)}=\frac{1}{X_{n}^{+}+X_{n}^{-}}\left(\begin{array}{cc}
\eta^{-1} X_{n}^{+}+\bar{\eta}^{-1} X_{n}^{-} & -\left(\eta^{-1}-\bar{\eta}^{-1}\right) Y_{n}^{+} \\
-\left(\eta^{-1}-\bar{\eta}^{-1}\right) Y_{n}^{+} & -\eta^{-1} X_{n}^{+}-\bar{\eta}^{-1} X_{n}^{-}
\end{array}\right),
$$

где

$$
\begin{aligned}
& X_{n}^{( \pm)}=(1 \pm i c \eta)^{n}(1 \mp i c \bar{\eta})^{n} \exp \left[\mp i c^{-1}\left(\frac{\eta}{1-\eta^{2}}-\frac{\bar{\eta}}{1-\bar{\eta}^{2}}\right) t\right], \\
& Y_{n}^{+}=(1 \pm i c \eta)^{n}(1 \pm i c \bar{\eta})^{n} \exp \left[\mp i c^{-1}\left(\frac{\eta}{1-\eta^{2}}+\frac{\bar{\eta}}{1-\bar{\eta}^{2}}\right) t\right] .
\end{aligned}
$$

При этом соответствующая матрица Дарбу имеет вид

$$
\begin{aligned}
D_{n}(\lambda) & =\lambda^{-1} I-\mathcal{H}_{n}^{(1)}= \\
& =\frac{1}{X_{n}^{+}+X_{n}^{-}}\left(\begin{array}{cc}
\lambda^{-1}\left(X_{n}^{+}+X_{n}^{-}\right)- & \left(\eta^{-1}-\bar{\eta}^{-1}\right) Y_{n}^{+} \\
-\left(\eta^{-1} X_{n}^{+}+\bar{\eta}^{-1} X_{n}^{-}\right) & \lambda^{-1}\left(X_{n}^{+}+X_{n}^{-}\right)+ \\
\left(\eta^{-1}-\bar{\eta}^{-1}\right) Y_{n}^{+} & +\left(\eta^{-1} X_{n}^{+}+\bar{\eta}^{-1} X_{n}^{-}\right)
\end{array}\right) .
\end{aligned}
$$

Подставляя (38) в (19), получаем в явном виде односолитонное решение

$$
U_{n}[1]=\left(\begin{array}{cc}
U_{n}^{+} & U_{n}^{-} \\
U_{n}^{-} & -U_{n}^{+}
\end{array}\right)
$$

где

$$
\begin{aligned}
& U_{n}^{+}=i c-\left(\eta^{-1}-\bar{\eta}^{-1}\right) \frac{\left(X_{n+1}^{+} X_{n}^{-}-X_{n+1}^{-} X_{n}^{+}\right)}{\left(X_{n+1}^{+}+X_{n+1}^{-}\right)\left(X_{n}^{+}+X_{n}^{-}\right)} \\
& U_{n}^{-}=\left(\eta^{-1}-\bar{\eta}^{-1}\right) \frac{Y_{n+1}^{+}\left(X_{n}^{+}+X_{n}^{-}\right)-Y_{n}^{+}\left(X_{n+1}^{+}+X_{n+1}^{-}\right)}{\left(X_{n+1}^{+}+X_{n+1}^{-}\right)\left(X_{n}^{+}+X_{n}^{-}\right)} .
\end{aligned}
$$

Решения приведены на рис. 1, 2. 


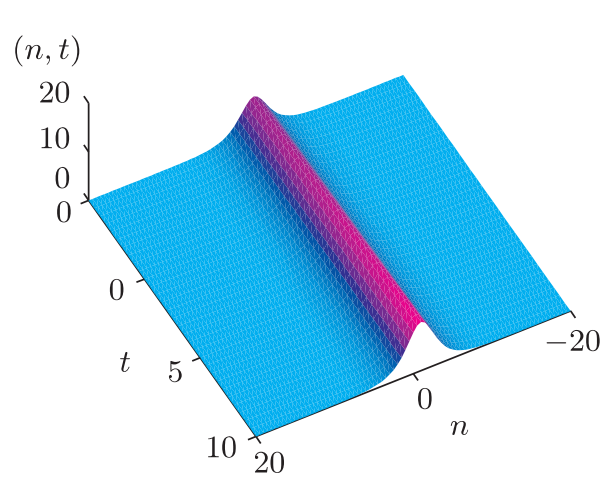

a

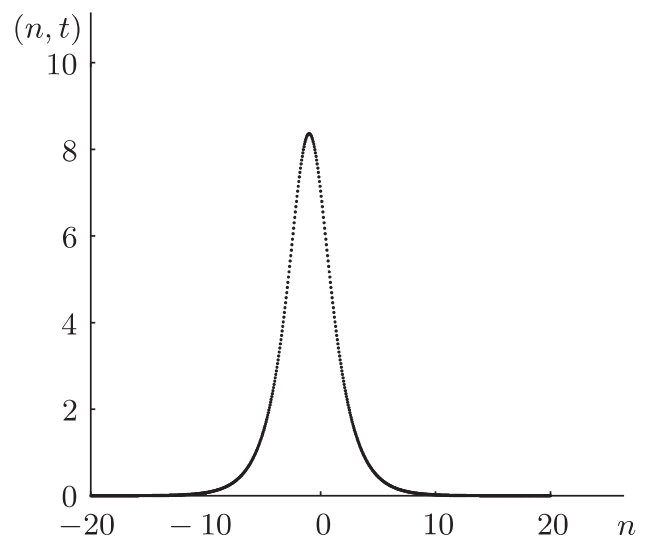

б

Рис. 2. Полудискретное односолитонное решение $U_{n}^{-}$; трехмерное представление (а) и двумерное представление (б) для численных значений $c=-6$, $\eta=0.049 i$.

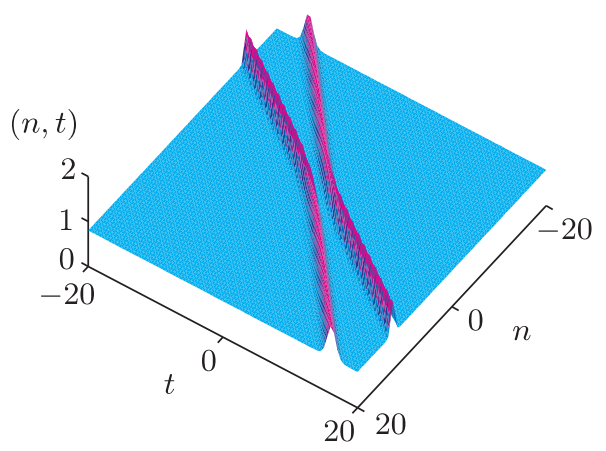

a

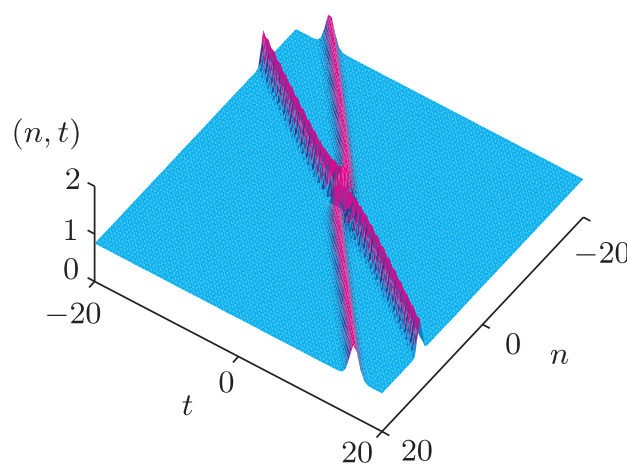

б

Рис. 3. Полудискретное двухсолитонное решение $U_{n}^{+}$; рассеяние (а) происходит при $c=0.6, \eta_{1}=0.8 i, \eta_{2}=-1.1 i$, а столкновение (б) - при $c=0.6$, $\eta_{1}=-0.8 i, \eta_{2}=-1.1 i$.

Скалярные функции $X_{n}^{ \pm}, Y_{n}^{+}$представлены выражениями (39). Исходя из двух наборов частных матричных решений $\mathcal{H}_{n, 1}$ при $\Lambda_{1}=\left(\begin{array}{cc}\eta_{1} & 0 \\ 0 & \bar{\eta}_{1}\end{array}\right)$ и $\mathcal{H}_{n, 2}$ при $\Lambda_{2}=\left(\begin{array}{cc}\eta_{2} & 0 \\ 0 & \bar{\eta}_{2}\end{array}\right)$ можно получить двухсолитонное решение с помощью формулы (21). Двухсолитонные решения приведены на рис. 3,4 .

Аналогично с помощью преобразования Дарбу можно найти явные выражения для многосолитонных решений модели ОМГР. 


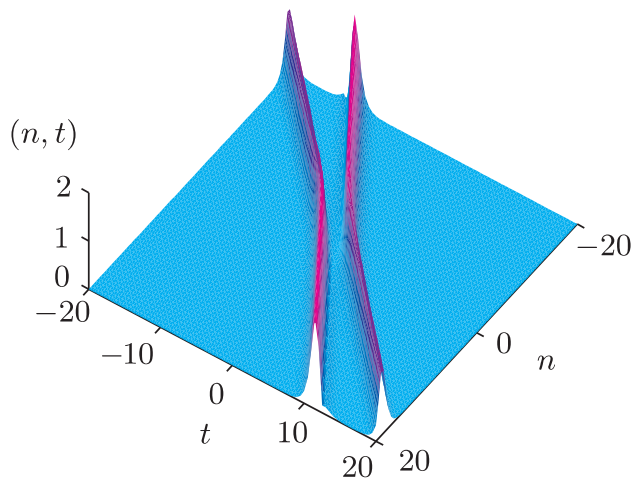

a

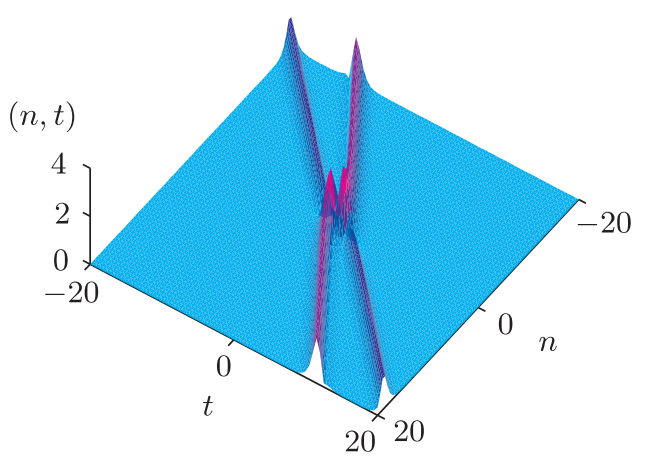

6

Рис. 4. Полудискретное двухсолитонное решение $U_{n}^{-}$; рассеяние (а) происходит при $c=-0.8, \eta_{1}=0.9 i, \eta_{2}=-0.5 i$, а столкновение (б) - при $c=-0.8$, $\eta_{1}=-0.9 i, \eta_{2}=-0.5 i$.

\section{6. ВЫВОДЫ}

В настоящей работе с помощью преобразования Дарбу изучаются квазидетерминантные многосолитонные решения модели ОМГР. Получено преобразование Дарбу на решениях пары Лакса. Затем решения выражены через квазидетерминанты. Наконец, в явном виде выведены многосолитонные решения для простого случая. Путем разложения квазидетерминантов получены также выражения для односолитонного решения. Приведены графические изображения одно- и двухсолитонных решений для полудискретной модели. Модель ОМГР для полностью дискретного случая будет рассмотрена в отдельной работе.

\section{Список литературы}

[1] L. A. Takhtajan, "Integration of the continuous Heisenberg spin chain through the inverse scattering method", Phys. Lett. A, 64:2 (1977), 235-237.

[2] Л. А. Тахтаджан, Л. Д. Фаддеев, Гамильтонов подход в теории солитонов, Наука, М., 1986.

[3] S. J. Orfanidis, "SU(n) Heisenberg spin chain", Phys. Lett. A, 75:4 (1980), 304-306.

[4] J. Honerkamp, "Gauge equivalence of exactly integrable field theoretic models", J. Math. Phys., 22:2 (1981), 277-281.

[5] L. D. Faddeev, "Integrable models in $(1+1)$-dimensional quantum field theory", Recent Advances in Field Theory and Statistical Mechanics (Les Houches, August 2-September 10, 1982), eds. J.-B. Zuber, R. Stora, North-Holland, Amsterdam, 1984, 561-608.

[6] Е. К. Склянин, "О некоторых алгебраических структурах, связанных с уравнением Янга-Бакстера", Функи. анализ и его прил., 16:4 (1982), 27-34.

[7] Y. Ishimori, "An integrable classical spin chain", J. Phys. Soc. Japan, 51:11 (1982), 3417-3418.

[8] F. D. M. Haldane, "Excitation spectrum of a generalized Heisenberg ferromagnet spin chain with arbitrary spin", J. Phys. C: Solid State Phys., 15:36 (1982), L1309-L1313.

[9] R. Balakrishnan, A. R. Bishop, "Nonlinear excitations on a ferromagnetic chain", Phys. Rev. Lett., 55:5 (1985), 537-540. 
[10] F.D. M. Haldane, "Geometrical interpretation of momentum and crystal momentum of classical and quantum ferromagnetic Heisenberg chains", Phys. Rev. Lett., 57:12 (1986), $1488-1491$.

[11] G. R. W. Quispel, F. W. Nijhoff, H. W. Capel, J. van der Linden, "Linear integral equations and nonlinear difference-difference equations", Phys. A, 125 (1984), 344-380.

[12] V.S. Gerdjikov, M. I. Ivanov, Y.S. Vaklev, "Gauge transformations and generating operators for the discrete Zakharov-Shabat system", Inverse Problems, 2:4 (1986), 413-432.

[13] N. Papanicolaou, "Complete integrabiblity for a discrete Heisenberg chain", J. Phys. A: Math. Gen., 20:12 (1987), 3637-3652.

[14] T. Tsuchida, "A systematic method for constructing time discretizations of integrable lattice systems: local equations of motion", J. Phys. A: Math. Theor., 43:41 (2010), 415202, $22 \mathrm{pp}$.

[15] A. Calini, "A note on a Bäcklund transformation for the continuous Heisenberg model", Phys. Lett. A, 203:5-6 (1995), 333-344.

[16] H. J. Shin, "Generalized Heisenberg ferromagnetic models via Hermitian symmetric spaces", J. Phys. A: Math. Gen., 34:14 (2001), 3169-3177.

[17] G. M. Pritula, V.E. Vekslerchik, "Stationary structures in two-dimensional continuous Heisenberg ferromagnetic spin system", J. Nonlinear Math. Phys., 10:3 (2003), 256-281.

[18] H. J. Shin, "SIT-NLS solitons in Hermitian symmetric spaces", J. Phys. A: Math. Gen., 39:15 (2006), 3921-3931.

[19] J.L. Cieśliński, J. Czarnecka, "The Darboux-Bäcklund transformation for the static 2-dimensional continuum Heisenberg chain", J. Phys. A: Math. Gen., 39:35 (2006), 11003-11012.

[20] O. Ragnisco, F. Zullo, "Continuous and discrete (classical) Heisenberg spin chain revisted", SIGMA, 3 (2007), 033, 6 pp., arXiv: nlin.SI/0701006.

[21] U. Saleem, M. Hassan, "Quasideterminant solutions of the generalized Heisenberg magnet model", J. Phys. A: Math. Theor., 43:4 (2010), 045204, 12 pp.

[22] M. Lakshmanan, "Continuum spin system as an exactly solvable dynamical system", Phys. Lett. A, 61:1 (1977), 53-54.

[23] V. B. Matveev, M. A. Salle, Darboux Transformations and Solitons, Springer, Berlin, 1991.

[24] C. Gu, H. Hu, Z. Zhou, Darboux Transformations in Integrable Systems. Theory and their Applications to Geometry, Mathematical Physics Studies, 26, Springer, Berlin, 2005.

[25] B. Haider, M. Hassan, "Quasi-Grammian solutions of the generalized coupled dispersionless integrable system", SIGMA, 8 (2012), 084, 15 pp., arXiv: 1211.1762.

[26] H. Wajahat A. Riaz, M. Hassan, "Darboux tramsformation of a semi-discrete coupled dispersionless integrable system", Commun. Nonlinear Sci. Numer. Simul., 48 (2017), 387-397.

[27] H. Wajahat A. Riaz, M. Hassan, "Multisoliton solutions of integrable discrete and semi-discrete principal chiral equations", Commun. Nonlinear Sci. Numer. Simul., 54 (2018), 416-427.

[28] И. М. Гельфанд, В. С. Ретах, “Детерминанты матриц над некоммутативными кольцами", Функи. анализ и его прил., 25:2 (1991), 13-25.

[29] P. Etingof, I. Gelfand, V. Retakh, "Nonabelian integrable systems, quasideterminants, and Marchenko lemma", Math. Res. Lett., 5:1-2 (1998), 1-12.

[30] I. Gelfand, S. Gelfand, V. Retakh, R. L. Wilson, "Quasideterminants", Adv. Math., 193:1 (2005), 56-141. 